\title{
DOE/ER/25120-1
}

Approximation of Attractors and Applications

Progress Report

for Period 5/1/92 to 10/31/92

ROGER TEMAM

INDIANA UNIVERSITY

Institute for Scientific Computing

\& Applied Mathematics

618 East Third Street \#108

Bloomington, Indiana 47405

October 23, 1992

Prepared for:

THE U.S. DEPARTMENT OF ENERGY

AGREEMENT NO. DE-FG02-92ER25120 


\section{Approximation of Attractors and Applications}

DOE Grant DE-FG02-92ER25120

$$
\text { May 1, } 1992 \text { - April 30, } 1993
$$

Principal Investigators: Roger Temam and Ciprian Foias

\section{Overview of the Project}

Our aim in this project is to explore what can be learned about turbulence from the theoretical and computational viewpoints, using the dynamical systems approach to turbulence.

According to Dynamical Systems theory the permanent regime for a turbulent flow is mathematically represented by an attractor that is a compact set in the phase space. It is hoped that the study of this attractor can improve our understanding of the physics of turbulent flows. It is also likely that the study of the approximation of this attractor can lead to efficient new algorithms for the numerical simulation of turbulence. Here some significant results have already been obtained, namely gain in computing time of up to $50 \%$ on actual CFD-Computational Fluid Dynamics-computations.

In general attractors are not differentiable manifolds. Therefore for computing purpose it is necessary to imbed them in, or approximate them by smooth manifolds.

An Inertial Manifold is a smooth manifold. It is finite-dimensional, it contains the attractor and attracts all orbits at an exponential rate. Hence the permanent regime takes place on it. From the physical viewpoint an inertial manifold is an interaction law between small and large eddies. When inertial manifolds are not known explicitly or do not exist, an interesting practical substitute is the Approximate Inertial Manifold.

The concept of Approximate Inertial Manifolds (AIM) yields simple smooth manifolds that approximate the attractor. By projecting the fluid mechanics equations (or similar dissipative evolution equations) on such manifolds we obtain a new type of numerical algorithms called the Nonlinear Galerkin methods. Several types of Nonlinear Galerkin meth- 
ods can be derived depending on the AIM which is used and on the discretization method used for the equation (spectral methods, finite elements, finite differences, wavelets). The utilization of the simplest AIM with a pseudo-spectral discretization has produced a very stable and efficient algorithm which allows reduction of computing time of about $50 \%$. In this project we intend to develop other forms of the Nonlinear Galerkin method which seem to be very promising. For instance in the case of finite difference discretizations this is done by using multigrid methods in conjunction with the newly introduced concept of incremestal unknowns (IU method).

A more complicated but probably more accurate way to identify the attractor is to approximate it by algebraic sets obtained by equating to zero explicit polynomials which are theoretically very small on the attractor. The corresponding algorithms confirm the theoretical results but are still time consuming and necessitate further substantial theoretical and numerical studies.

On the theoretical side we intend to study more particularly the equations of n. eorology and oceanography and to study the connection between inertial manifolds and the concept of slow manifolds of meteorology which is a particular case of inertial manifold.

\section{Progress Report}

\section{During the first six months two directions of research have been explored} as part of this project:

(a) Study of Dynamical Systems relat u to dissipative evolution equations, in particular the incompressible Navier-Stokes equations.

Attractors and Inertial Manifolds and their approximation.

Application to the development of computationally efficient numerical methods for the long time approximation of these equations (in particular Nonlinear Galerkin Method, Incremental Unknowns Method). 
(b) Mathematical formulation of models of coupled Atmosphere and Ocean (CAO), based on the primitive equations of the atmosphere and the ocean.

Study of these equations as dynamical systems.

Beside these research topics, a book by C. Foias, O. Manley and R. Temam provisionally entitled "Turbulence and Navier-Stokes equations" is in preparation.

For direction (a) the current research, and the results completed include the following

- Existence of Inertial Form (Inertial Manifold) for the two-dimensional Navier-Stokes equations on the sphere (Temam-Wang, see [TW]). Also we have obtained for the first time an estimate of the dimension of the inertial manifold in terms of the Grashof number (that bound is high, but still it is polynomial with respect to the Grashof number).

- Construction of Approximate Inertial Manifolds of higher order for modelling of turbulence (Foias, Temam in collaboration with Oscar Manley, see [FMT2]).

Here a method for the elimination (or averaging) of the fine structure of fluid turbulence was developed by constructing appropriate approximate inertial manifolds. The method depends in part on recognizing that in turbulent flows the high wave number modes contain a negligible portion of the total energy in the velocity field. This permits successive suppression of those modes by an iterative method, yieldirig a sequence of approximate inertial manifolds with a decreasing number of degrees of freedom, and a corresponding decrease in the needed computational resources. It was shown that the method leads to an effective viscosity which increases monotonically as more and more of the high wave number modes are eliminated (or effectively averaged out). In the limit of large, but finite Reynolds numbers the effective viscosity differs somewhat from that obtained by more conventional methods. At the same time the present approach has yielded an a priori estimate of the upper bound on the difference between the usually unavailable exact solution to the 
given fluid flow problem and the solution to the corresponding equations constituting the approximate inertial manifold. That is in distinct contrast with the more ad hoc intuitive methods for approximate solutions of Navier-Stokes equations at high Reynolds numbers, where no such error estimates are possible. In practice it will enable a trade off between the extent of the maximum tolerable error in direct numerical simulations and the amount of fine detail retained in calculating the given velocity field.

- Development of the Nonlinear Galerkin Method .

The Nonlinear Galerkin method is a multilevel method where high and low frequency components of the flow are treated differently (in relation with the Approximate Inertial Manifolds (AIMs)). The method was previously implemented for the 2D and 3D NavierStokes equations in the space periodic case (homogeneous flows) using pseudo-spectral discretizations, adaptative procedures (change of levels), and the simplest nonflat AIM, i.e. the one introduced by Foias, Manley and Temam in [FMT1].

We now study theoretical and computational problems related to its implementation in nonhomogeneous flows, using Tschebychev polynomials and collocation methods ( $J$. Shen, R. Temam). T. Dubois has developed a computational CFD code (Navier-Stokes $2 \mathrm{D}$ and $3 \mathrm{D})$ for its implementation. The first numerical results were presented at the 13 th International Conference on Numerical Methods in Fluid Dynamics ICNMFD, Rome, July 1992.

Much wcrk remains to be done in this direction of research. It is important as it makes the connection between theory and actual computations. We intend to pursue these efforts further.

For direction (b) the current research, and the results completed include the following:

- In a previous work we have completed two articles providing for the first time a 
rigorous mathematical formulation of the primitive equations of the atmosphere and of the ocean (Temam, Wang in collaboration with J. L. Lions), see [LTW1,2].

- At this time we study models of coupled atmosphere and ocean (CAO) based on the primitive equations. The following questions will be addressed (see [LTW3]):

\author{
Modeling of $\mathrm{CAO}$ \\ Mathematical Theory \\ Numerical Analysis of the CAO models.
}

\title{
References
}

[FMT1] Foias, C., Manley, O., and Temam, R., Modelling of the interaction of small and large eddies in two dimensional turbulent flows, Math. Model and Numer. Analysis (M2AN), 22, (1988), 93-114.

[FMT2] Foias, C., Manley, O., and Temam, R., Iterated Approximate inertial manifolds for Navier-Stokes equations in 2D, J. Math. Anal. Appl., to appear.

[LTWi] Lions, J.L., Temam, R. and Wang, S., New formulations of the primitive equations of the atmosphere and applications, Nonlinearity, 5, (1992), p. 237288.

[LTW2] Lions, J.L., Temam, R. and Wang, S., On the equations of the large-scale ocean, Nonlinearity, 5, (1992), 1007-1053.

[LTW3] Lions, J.L., Temam, R. and Wang, S. Models of the coupled atmosphere and ocean (CAO I), in preparation.

[TW] Temam, R. and Wang, S., Inertial forms of Navier-Stokes equations on the sphere, submitted to J. Funct. Analysis.

\section{DISCLAIMER}

This report was prepared as an account of work sponsored by an agency of the United States Government. Neither the United States Government nor any agency thereof, nor any of their employees, makes any warranty, express or implied, or assumes any legal liability or responsibility for the accuracy, completeness, or usefulness of any information, apparatus, product, or process disclosed, or represents that its use would not infringe privately owned rights. Reference herein to any specific commercial product, process, or service by trade name, trademark, manufacturer, or otherwise does not necessarily constitute or imply its endorsement, recommendation, or favoring by the United States Government or any agency thereof. The views and opinions of authors expressed herein do not necessarily state or reflect those of the United States Government or any agency thereof.

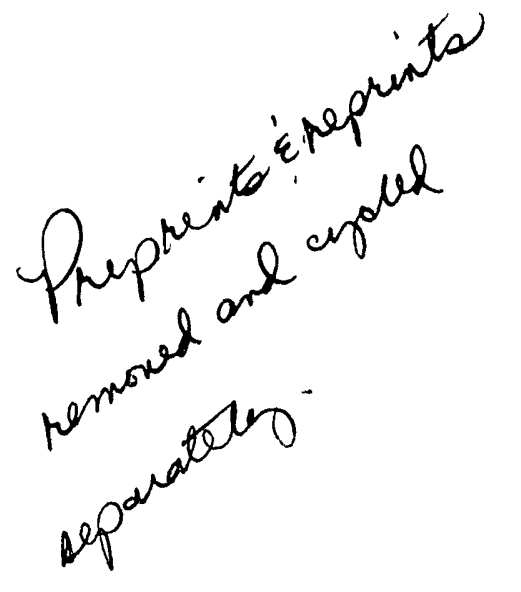



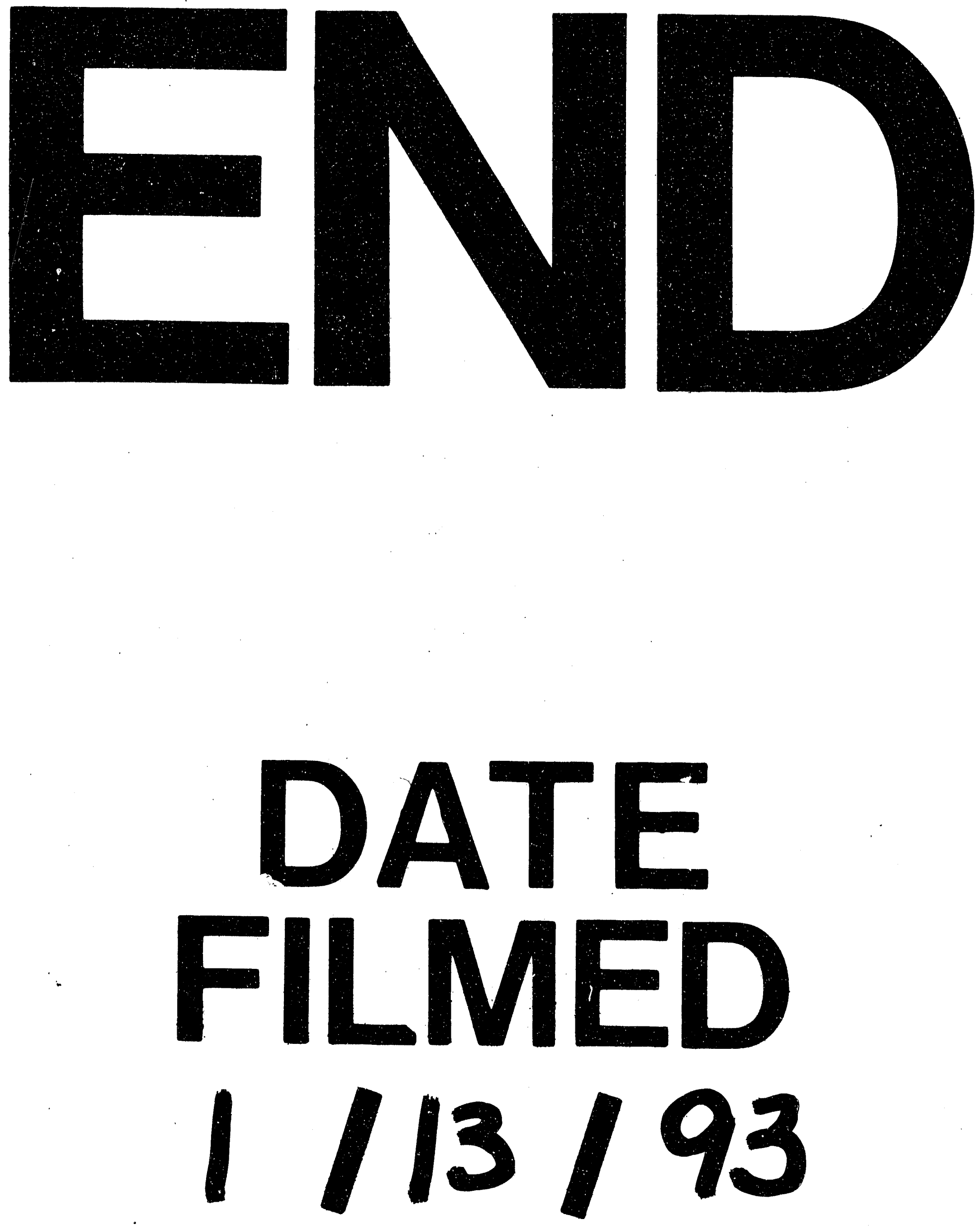
